T1D are needed, particularly in teenagers, and structured education on managing alcohol consumption may need revision.

\section{OP47 THE IMPACT OF MAJOR MENTAL ILLNESS ON QUALITY OF CARE IN PEOPLE WITH TYPE 2 DIABETES IN SCOTLAND: AN ANALYSIS OF ROUTINELY COLLECTED HEALTH DATA}

\begin{abstract}
${ }^{1,4} \mathrm{KJ}$ Fleetwood* ${ }^{*},{ }^{1,4} \mathrm{SH}$ Wild, ${ }^{2} \mathrm{DJ}$ Smith, ${ }^{3} \mathrm{~K}$ Licence, ${ }^{1} \mathrm{SW}$ Mercer, ${ }^{1} \mathrm{C}$ Sudlow, ${ }^{1,4} \mathrm{C}$ Jackson. ${ }^{1}$ Usher Institute of Population Health Sciences and Informatics, The University of Edinburgh, Edinburgh, UK; ${ }^{2}$ Institute of Health and Wellbeing, University of Glasgow, Glasgow, UK; ${ }^{3}$ Public Health and Intelligence, NHS National Services Scotland, Edinburgh, UK; ${ }^{4}$ Edinburgh Medical School, University of Edinburgh, Edinburgh, UK
\end{abstract}

\subsection{6/jech-2019-SSMabstracts.48}

Background Recent evidence from some countries suggests that people with a mental health condition receive poorer type 2 diabetes mellitus (T2DM) care than people without a mental health condition. We aimed to investigate whether history of a major mental illness affects quality of care in people with T2DM in Scotland.

Methods We identified adults diagnosed with T2DM between 2007 and 2015 from a 2016 extract of Scotland's national diabetes register (the Scottish Care Information (SCI) - Diabetes database). We used International Classification of Disease codes to identify history of mental illness from pseudonymously linked Scottish psychiatric and acute hospital admission records. Retinopathy screening and HbA1c measurement within the first year post T2DM diagnosis were determined from the diabetes register. Using logistic regression analysis, we obtained odds ratios (ORs) for receipt of both tests for people with a history of schizophrenia, bipolar disorder or depression in hospital records, compared to those without a history of mental illness in hospital records.

Results We included 129,028 people with T2DM. Of these, 1,457 (1.1\%) had schizophrenia, $653(0.5 \%)$ had bipolar disorder and 4,132 (3.2\%) had depression. Within the first year post T2DM diagnosis, $84.1 \%$ of the cohort received retinopathy screening and $92.5 \%$ received $\mathrm{HbA} 1 \mathrm{c}$ measurement. Both retinopathy screening and $\mathrm{HbA} 1 \mathrm{c}$ measurement were received by $81.3 \%$ of people without a history of mental illness compared to $75.0 \%$ of people with schizophrenia, $77.5 \%$ of people with bipolar disorder and $77.7 \%$ of people with depression. After adjusting for health board, year, age, sex, area-based deprivation, ethnicity and comorbidities, the odds of receiving both tests were lower in people with schizophrenia (OR 0.77, 95\% confidence interval (CI) 0.68, 0.87), bipolar disorder (OR 0.78, 95\% CI 0.65, 0.94) and depression (OR $0.82,95 \%$ CI $0.76,0.89$ ) compared to those without a history of mental illness. These differences were driven by lower percentages of retinopathy screening amongst people with schizophrenia, bipolar disorder or depression; proportions with HbA1c measurement were similar across all groups.

Conclusion Compared to people without a history of mental illness, people with schizophrenia, bipolar disorder or depression are less likely to receive diabetic retinopathy screening within the first year post T2DM diagnosis. Such discrepancies in care may contribute to poorer T2DM outcomes amongst people with a major mental illness. Further work will investigate whether discrepancies in care persist beyond the first year post T2DM diagnosis and how discrepancies in care have evolved over time.

\section{OP48 THE PREVALENCE AND RISK FACTORS OF POLYPHARMACY AMONG DIABETIC PEOPLE: EVIDENCE FROM THE ENGLISH LONGITUDINAL STUDY OF AGEING (ELSA)}

${ }^{1} Y \mathrm{~T}$ Huang, ${ }^{2} \mathrm{~L}$ Wei, ${ }^{1} \mathrm{~A}$ Steptoe, ${ }^{1} \mathrm{P}$ Zaninotto* ${ }^{1}$ Epidemiology and Public Health, University College London, London, UK; ${ }^{2}$ School of Pharmacy, University College London, London, UK

\subsection{6/jech-2019-SSMabstracts.49}

Background Diabetes among older people is becoming more common worldwide. Polypharmacy is an important issue among older people with multimorbidity; however, relevant studies focusing on older people with diabetes are scarce. Therefore, the role of polypharmacy in this vulnerable population remains uncertain. The aim of this study is to investigate the prevalence of polypharmacy among older people with and without diabetes, and to determine the potential risk factors for polypharmacy.

Methods A nationally representative cross-sectional study, ELSA 2012/2013, was used and 7729 participants aged 50109 were investigated. Polypharmacy was defined as taking five to nine long-term used medications daily for chronic diseases or chronic symptoms, while using ten or more medications was categorised as excessive polypharmacy. The presence of illness was defined as either self-reported diagnosis or being prescribed specific medications for the condition. The number of comorbidities was generated based on the combined diagnoses excluding diabetes. Multinomial logistic regression was applied to estimate risk factors for polypharmacy, and potential social determinants were also included.

Results The prevalence of polypharmacy was $21.4 \%$ in 2012 , and only 3\% was excessive polypharmacy. $51.6 \%$ of people with type 2 diabetes reported polypharmacy and 10.2\% excessive polypharmacy. These rates were significantly higher than the $16.4 \%$ polypharmacy and $1.8 \%$ excessive polypharmacy among people without diabetes $(\mathrm{p}<0.001) .74 .6 \%$ diabetic people had three or more comorbidities, compared with $40.8 \%$ in people without diabetes $(\mathrm{p}<0.001)$. Among people with three or more comorbidities, polypharmacy was present in $61.5 \%$ of people with diabetes, compared with $36.0 \%$ in people without diabetes. Significant risk factors for polypharmacy were diabetes (Relative-risk ratios/RRR $=4.06$, 95\% CI $3.38,4.86)$, older age $(\mathrm{RRR}=1.02,95 \%$ CI $1.01,1.03)$, male $(\mathrm{RRR}=0.64,95 \%$ CI $0.55,0.75)$, more comorbidity $(\mathrm{RRR}=2.46$, 95\% CI 2.30, 2.62), living with a partner $(\mathrm{RRR}=1.20,95 \% \quad \mathrm{CI} 1.01,1.42)$, and less wealth $(\mathrm{RRR}=0.93$, 95\% CI 0.87, 0.98). However, age, living with partner, and wealth were not significantly related to excessive polypharmacy. Diabetes and the number of comorbidities were predominant risk factors for excessive polypharmacy.

Conclusion Polypharmacy was a prevalent phenomenon in the English older population, and it was more severe in people with diabetes. The presence of diabetes and having comorbidities were the main contributors to polypharmacy and excessive polypharmacy after adjusting for important covariates. Current evidences confirm both health condition and socioeconomic status are associated with medication use. This research 